\title{
Instrumentation for the Thirty Meter Telescope
}

\section{Keith Taylor}

Keith Taylor, "Instrumentation for the Thirty Meter Telescope," Proc. SPIE 5492, Ground-based Instrumentation for Astronomy, (30 September 2004); doi: $10.1117 / 12.556885$

EDent: SPIE Astronomical Telescopes + Instrumentation, 2004, Glasgow, United Kingdom 


\title{
Instrumentation for the Thirty Meter Telescope
}

\author{
K. Taylor* \\ California Institute of Technology, Department of Astronomy, 105-24 Caltech, \\ 1201 East California Blvd, Pasadena CA 91125, USA
}

\begin{abstract}
The Science Advisory Committee (SAC) of the Thirty Meter Telescope Project (TMT) is nearing completion in its deliberations on defining a set of instrumentation capabilities for the first decade of operation. These are encapsulated in the SAC's Science Requirements Document (SRD). We focus here on issues related to the challenges posed by the proposed first generation requirements for both seeing-limited, wide-field spectroscopy for the UV/optical and for integral field unit (IFU) spectroscopy for the near infra-red (NIR).
\end{abstract}

Keywords: ELT, Instrumentation, TMT, IFU, Fibers, Slicers

\section{Introduction}

The TMT SAC has defined its first generation optical and NIR instrumentation capabilities [1] as follows:

- Diffraction-limited, moderate spectral resolution 1 to $5 \mu \mathrm{m}$ spectroscopy (utilizing slits or IFUs) over small fields; and, with lower spatial resolution over a more limited, 0.8 to $2.5 \mu \mathrm{m}$ wavelength range, multi-object, IFU-based spectroscopy over a 5 arcmin field. (The desire to combine these two capabilities is based on the somewhat unsafe assumption that the $\mathrm{AO}$ system will be common to both; however we will consider these as separate instruments for the purposes of the following discussion.)

- Seeing-limited massively multiplexed $150<\mathrm{R}<6000$ spectroscopy in the 0.31 to $1 \mu \mathrm{m}$ wavelength range over a wide (10-20 arcmin) field.

A diffraction-limited, high-spectral-resolution mid-IR spectroscopic capability has also been defined but will not be discussed here.

This paper will address some of the strategies that are being considered by members of the TMT community - as coordinated by its Instrument Working Group $\left(\mathrm{IWG}^{1}\right)$ - in order to facilitate these demanding instrumental requirements and hence summarizes the work of several individuals who are working to define the TMT's initial instrumentation package. A special urgency has been placed on defining base-line designs from the project's desire to understand the constraints that might be placed on the telescope's optical configuration or structural design by what are anticipated to be massive, complex and voluminous instruments.

\section{Small Field Diffraction-Limited NIR Imager and Spectrometer}

The primary capability envisaged here is for a small field ( $\sim 2$ arcsec.), on-axis, NIR spectroscopy at the diffraction limit of the TMT. A secondary goal would be to supply diffraction-limited imaging over relatively broad NIR wavelength bands over a $\sim 10$ arcsec. field of view. While specifications for the adaptive optics (AO) system are defined in the SRD, no attempt has been made to align these with anticipated (or real-life) AO systems. Given the relative immaturity of such AO systems for ELTs, the project has chosen to define scientific requirements rather than to second-guess the

\footnotetext{
${ }^{1}$ IWG members: D.Crampton [Chair] \& G.Walker (Canada); D.Simons \& D.Sprayberry (AURA); S.Vogt \& J.Larkin (UC); K.Matthews \& K.Taylor (Caltech)

* kt@astro.caltech.edu, phone:+1 (626) 395-8488
} 
AO technology. In this case however, such narrow-field requirements are expected to be safely within AO performance expectations.

\section{Requirements (and Goals) summary:}

- Wavelength range $(\Delta \lambda)$ : Requirement: $\Delta \lambda \sim 0.8-2.5 \mu \mathrm{m}$; (Goal: $\Delta \lambda \sim 0.6-5.0 \mu \mathrm{m})$;

- $\quad$ Field of view (FoV):

- $\quad<2$ arcsec. (IFU spectroscopy);

- $\quad<10$ arcsec. (imaging);

- $\quad$ Spatial sampling approaching Nyquist sampling. For example:

- $\quad$ Spectroscopy at $\lambda \sim 2 \mu \mathrm{m}$ implies $\sim 7$ mas sampling capable of producing $\sim 10^{5}$ individual spectra;

- $\quad$ Imaging at $\lambda \sim 1 \mu \mathrm{m}$ implies $\sim 3.5$ mas sampling over a $3 \mathrm{k}^{2}$ pixel detector;

- $\quad$ Spectral Resolution $(\boldsymbol{R})$ :

- $\quad$ Spectroscopy: $\boldsymbol{R} \sim 4,000$ over entire J, H, K and L bands (one band at the time);

- Imaging: $\boldsymbol{R} \sim 2$ to 50 for imaging mode.

It should be noted that such a spectroscopic system requires a raw pixel count equivalent to $\sim 64$ spectrographs each feeding a $2 k^{2} \mathrm{HgCdTe}$ array which, assuming current prices, would imply a budget of $\sim \$ 30 M$ in detectors alone.

For scaling purposes we assume Nyquist sampling at the image plane with either spectroscopic input apertures (eg: fibers or slicers [2]) or detector pixels for the imaging case. For both imaging and spectroscopy, the sample size for a given wavelength, $\lambda$, is given simply by $\lambda . f_{\mathrm{r}} / 2.2$ where $\mathrm{f}_{\mathrm{r}}$ is the incident focal ratio. For spectroscopy, this places stringent constraints on both fiber and slicer systems.

Fiber systems: In order to mitigate focal-ratio degradation (FRD), fibers are best operated at f-ratios $\left(f_{r}\right)$ near their numerical aperture (NA). If we assume a modestly slow canonical fiber input f-ratio of $\mathrm{f} / 5$ then a fiber diameter of $\sim 2.5 \mu \mathrm{m}$ is implied at a wavelength $\lambda \sim 1 \mu \mathrm{m}$. Multi-mode fibers are generally $>10$ times this size implying a highly nonoptimized fiber spectrograph design which is forced to sacrifice camera speed and beam size. Furthermore, the requirement to spatially resolve each individual fiber spectra generally implies an inefficient usage of detector real estate, requiring factors of $\sim 2$ or more pixels than an equivalent slit or slicer system. This is particularly critical for IFU systems which are already very pixel hungry given the spatio-spectral (3D) nature of their raw data product.

To example such a system in practice we will make some working assumptions:

- $\quad$ Fiber core $=50 \mu \mathrm{m}$ (near-field uniform illumination at output);

- $\quad$ Output fiber f-ratio: $\mathrm{f}_{\mathrm{r}}^{\text {OUT }} \sim 5$;

- $\quad$ Detector Nyquist ( 2 pixel) sampling with $9 \mu$ m pixels.

This latter assumption presupposes a development in the manufacture of small pixel NIR detectors which would have the potential for improved noise (both read and dark) critical to diffraction-limited, intermediate resolution spectroscopy. Such assumptions straightforwardly imply camera f-ratios faster than $\sim \mathrm{f} / 2$ with slit-width resolution products down by a factor of $\sim 3.5$ with respect to a comparable slicer system.

Such arguments are not fatal and are not to be taken as excluding fiber systems from considerations, however they do indicate that other approaches need to be carefully considered and qualitatively compared.

Slicer systems: In this case the same scaling arguments, combined with the constraint that slice thicknesses are currently limited to $>300 \mu \mathrm{m}$ for ease of fabrication and assembly, imply incident f-ratios slower than $\mathrm{f}_{\mathrm{r}}>660$ placing awkward constraints on their optical design and alignment. Furthermore, through imaging the sliced slit directly onto the detector, diffraction-limited slicer systems are required to stay within the defined wave-front error budget from magnification optics through slicer to the detector. This issue is less challenging for fiber systems that intersect the delivered wavefront at the input of the fiber (or lenslet/fiber) interface and thereon each wavefront sample is treated independently. 


\section{Multi-Object Intermediate Resolution, Near-IR Spectrometer}

This instrument is envisioned to work behind a wide field AO system (often referred to, in this context, as multi-object $\mathrm{AO}$ or MOAO) that delivers individually corrected small fields over a large $(\sim 5 \operatorname{arcmin})$ patrol field of view. Each small field ( $\leq 2$ arcsec) deployable IFU (or d-IFU) will have its own AO deformable mirror (DM) and will feed the spatially resolved information into multiple spectrographs. Given that this capability is specific to the study of multiple extended objects, diffraction limited spatial resolution is not required, rather a $\sim 50$ mas spatial sampling is considered adequate.

\section{Requirements summary:}

- Wavelength range: $\Delta \lambda \sim 0.8-2.5 \mu \mathrm{m}$;

- Patrol field of view $\sim 5$ arcmin.;

- Deployable IFU specifications:

- $\quad$ Field of view $<2$ arcsec.;

- Spatial sampling $\sim 50$ mas;

- $\quad$ IFU object multiplex $>10$;

- Closest required nearest-neighbor distance $\sim 20$ arcsec.;

- Spectral Resolution $(\boldsymbol{R})$ :

- $\quad \boldsymbol{R} \sim 2,000$ to 10,000 over entire J, H, K and L bands (one band at the time).

It will be noted that such a system requires, at maximum, a single $2 k^{2} H g C d T e$ spectrograph for each IFU and hence places a much smaller demand on its pixel budget than the diffraction-limited IFU system described earlier.

For scaling purposes we again assume Nyquist sampling at the image plane with fibers or slicers forming the spectroscopic input apertures.

Fiber systems: A 50 $\mu \mathrm{m}$ fiber core will subtend 50 mas at an input f-ratio, $\mathrm{f}_{\mathrm{r}}^{\mathrm{IN}} \sim 7$ whose FRD will imply an $\mathrm{f}_{\mathrm{r}}^{\text {OUT }} \sim 5$. Such a spectrograph will require modest camera speeds of $\sim \mathrm{f} / 4$ in order to be Nyquist sampled on an $18 \mu \mathrm{m}$ pixels detector. (NB: For such spatio-spectral resolutions, sky flux between the $\mathrm{OH}$ lines will dominate detector noise for typical exposure times and hence there is less of an argument for small pixels $\mathrm{HgCdTe}$ detectors in this case.) Nevertheless, the inevitable loss in luminosite (A $\Omega$ ) created by the combination of FRD and lenslet/fiber coupling [3] will compromise performance and/or spectrograph cost. Furthermore, the non-contiguous nature of fibers as formatted onto the detector requires a factor of $\sim 2$ loss in efficiency in the number of pixels required per resolved spatio-spectral element.

None of these disadvantages are fatal to such a fiber system while the advantages of fibers for use in a multi-object capacity are well known and documented and the use of fibers in the near-IR and for cryogenic systems has been extensively discussed and prototyped [4].

Slicer systems: In this case the same scaling arguments lead to fairly relaxed constraints conducive to a wide range of slicer designs with slicers thicknesses in the comfortable $\sim 500 \mu \mathrm{m}$ to $\sim 2 \mathrm{~mm}$ range. Also, for this non-diffraction limited (50mas sampling) case, the constraints on wave-front aberration are significantly relaxed and arguments for sampling the wave-front early with lenslet/fibers are substantially less compelling. Nevertheless the attraction of fibers becomes an issue when the ease or otherwise of multi-IFU deployment is considered. A slice -vs- fiber comparison must be judged on the basis of a detailed full system analysis of all options which fold in the design of deployment mechanisms, the DM location and size, magnification fore-optics, spectrograph design and cryogenic considerations. The TMT project is only just begin to contemplate such a trade study.

d-IFU Deployment schemes: Fiber systems are unique in allowing for what are referred to as "AO button" concepts where the button contains not only the DM but also the magnification fore-optics and the input lenslet/fiber feed. Fiber are then routed from each button to an array of remotely located cryogenic spectrographs. Optical designs for such buttons are being explored by several TMT groups in order to ascertain dimensions and practicabilities for deployment across the telescope's focal surface. 
More traditional approaches to IFU deployment for both fiber and slicer systems are also being studied. In particular, the pick-off mirror concept as pioneered by the UK/ATC's GIRMOS team [5] and as proposed for the VLT's KMOS-1 system has great appeal, while the KMOS-2 concept [6] from the Laboratoire d'Astrophysique de Marseille also has considerable merit. No doubt other systems involving, for example, StarBugs [7], should be evaluated within the TMT context. Special consideration needs to be given to the scale of the MOAO focal plane (dia. $\sim 600 \mathrm{~mm}$ for an $\mathrm{f} / 15$ telescope) and the detailed interpretation of the $\sim 20$ arcsec nearest neighbor requirement before a proper comparison can be made. Furthermore, given the cryogenic requirements of the spectrographs, great care will need to be taken in minimizing cryogenic mechanisms for what promises to be a very complex, many-motor, system.

\section{Wide Field Optical Imaging Spectrometer}

This instrument (WFOS) is specified to collect seeing-limited, multi-object spectra over a large field comparable with the nominal 20 arcmin. field diameter delivered at the secondary focus of the TMT. The instrumental performance should be optimized for the full UV/optical window as defined by the atmospheric cut-off at $310 \mathrm{~nm}$ out to the CCD sensitivity limit beyond $1.0 \mu \mathrm{m}$. While the goal is to record the entire wavelength range in a single exposure, it is recognized that, for optimized performance, this wavelength range may be split thus requiring two or more exposure utilizing optimized arms for each wavelength range. Preliminary investigations have revealed great difficulty in approaching the $200 \mathrm{arcmin}^{2}$ inscribed square field, let alone the full $300 \mathrm{arcmin}^{2}$ circular field which could only be practically achieved with a multi-object fiber instrument like $2 \mathrm{dF}$ [8]. However, the demand for maximal single object efficiency, competitive in throughput with the front-ranked multi-slit spectrographs (eg: DEIMOS; LRIS; VIMOS, GIMOS), predisposes WFOS to be a multi-slit instrument and hence a more pragmatic field of view requirement has been specified at $75 \operatorname{arcmin}^{2}$. This field need not be contiguous but can be dissected into multiple spectrographs if necessary.

\section{Requirements (and Goals) summary:}

- Wavelength range: Requirement: $\Delta \lambda \sim 0.31-1.0 \mu \mathrm{m}$; (Goal: $\Delta \lambda \sim 0.30-1.3 \mu \mathrm{m})$;

- Field of view: Requirement: $\sim 75 \operatorname{arcmin}^{2}$ (Goal: $300 \operatorname{arcmin}^{2}$ );

- $\quad$ Total slit length $>500$ arcsec (implying a multi-object capacity, $\mathrm{M}_{\#}>50$ );

- Image quality; including telescope and Atmospheric Dispersion Corrector (ADC) $<0.2 \operatorname{arcsec}(\mathrm{FWHM})$ over a band-width, $\Delta \lambda \sim 100 \mathrm{~nm}$ throughout the sensitivity range;

- Slit-width/resolution product: Requirement: 3750 arcsec (Goal: $4500 \operatorname{arcsec}$ ); Spectrograph throughput $>30 \%$ (telescope focus to detector);

- Desirable features:

- $\quad$ Cross-dispersed mode for modest $\mathrm{M}_{\#}$

- Imaging through narrow-band filters

- $\quad$ Possible use of d-IFUs.

Early in the TMT project's gestation phase WFOS was recognized as a capability which could potentially drive aspects of telescope optical design and configuration; hence some urgency was placed on deriving conceptual designs which might satisfy the SAC's requirements. As a result, three competitive studies were launched as a means of probing a sufficient volume of design space to allow some security in the conclusions reached. The three studies are identified as follows:

- $\quad$ ELVIS: Harland Epps \& Joe Miller (University of California at Santa Cruz) [9]

- CIT-WFOS: Keith Taylor (Caltech) \& Damien Jones (Prime Optics Inc.)

- HIA-WFOS: Bev Oke, Ian Powell \& Denis Laurin (Herzberg Institute of Astrophysics)

Fortuitously all three programs took quite separate approaches to the problem and hence the exercise served well in its goal of exploring multiple design spaces. UCSC's ELVIS concept used a naked f/15 Nasmyth focus to deliver a single, contiguous field, spectrograph while both the CIT and HIA WFOSs employed focal reducers to bring the linear dimensions of the input focal plane to manageable proportions. The CIT-WFOS came close to utilizing the full 200 
$\operatorname{arcmin}^{2}$ inscribed square field with a single spectrograph while the HIA-WFOS segmented the field into 4 separate smaller field spectrographs each with its own focal reducer. A brief description of ELVIS and the CIT-WFOS follows: the HIA system is the subject of a separate SPIE paper [10] to appear in the same proceedings and hence will not be discussed further here.

ELVIS: As stated previously, ELVIS accepts a naked f/15 Nasmyth focus but requires an Aplanatic Gregorian (A-G) telescope configuration to achieve its wide field. ELVIS assumes a trombone-type ADC ahead of the telescope image surface and utilizes fully refractive collimators and cameras whose dimensions are constrained to the size of currently available $\mathrm{CaF}_{2}$ blanks. The collimator forms a $250 \mathrm{~mm}$ beam which limits the achievable slit-width/resolution product to somewhat below specification for efficient operation, however it is designed with three wavelength optimized arms which can be selected independently with the option of using two arms simultaneously through the use of a suitably large dichroic. The imaging and spectroscopic field of view is limited by the collimator to a diameter of $\sim 8$ arcmin. (50 $\operatorname{arcmin}^{2}$ ) in a single contiguous field while the $\mathrm{f} / 2.3$ camera achieves a massive $\pm 0.24^{\mathrm{c}}$ field through the use of VPH transmission gratings close to its front element. ELVIS thus requires articulation of its cameras to achieve blaze and dispersion selection for a given grating. It should be noted that a nominal 0.75 arcsec slit will subtend $\sim 17$ pixels $(15 \mu \mathrm{m}$ pitch) with the full camera field servicing a 150 Mpixel CCD array. While the concept falls somewhat short of the formal requirements for field of view and spectral resolution, nevertheless it has the merit of being buildable with currently available technologies and materials and thus represents a relatively low risk option.

CIT-WFOS: In contrast, Caltech's concept derives from the earlier three-mirror focal reducer design (3MfR) for the CELT project which attempted to maximize field of view free from detailed considerations of cost and risk. The CITWFOS relays the naked $\mathrm{f} / 15$ Nasmyth to an intermediate $\mathrm{f} / 5$ focus using an off-axis, three-mirror design. Like ELVIS it prefers an A-G configuration but can work with Ritchey-Chretien (R-C) if necessary. The f/5 image surface allows a much reduced size for the ADC and the slit masks while the focal reducer delivers a pupil-centric field which is ideal for a d-IFU feed. While reflection losses in the three-mirror focal reducer may be contained to within $\sim 15 \%$ through use of recently developed multi-layer reflective coatings nevertheless such losses are serious for an instrument with such demanding throughput specifications. However, in this case, these losses may be more than compensated by the use of a Ground Layer Adaptive Optics (GLAO) mirror located on the second focal reducer mirror; a 500mm mirror coincident with the telescope pupil.

A single spectrograph follows the focal reducer and ADC. It has a refractive collimator, optimally matched to the field curvature of the $3 \mathrm{MfR}$, which delivers a $500 \mathrm{~mm}$ beam for adequate spectral resolution. This is followed by a very fast catadioptric, $\mathrm{f} / 0.8$, camera facilitated through use of CCDs which are curved to match the focal surface of the camera itself; the avoidance of a field-flattener de-constrains the optimization of such fast cameras making them a very attractive option for pixel-matching on ELTs. Such a system can, in principle deliver a very large, $175 \operatorname{arcmin}^{2}$ field of view however its practicality, cost and risk have yet to be evaluated.

\section{CONCLUSIONS}

Lead by the TMT's Instrumentation Working Group, several of the first generation instrumentation capabilities, identified by the Science Advisory Committee, are being studied in part with a view to elucidating their impact on critical optical and configurational design issues for the telescope. Key concepts and technologies are being identified for the NIR IFU capabilities while strong constraints, in particular, on the telescope optical design are being defined by the need for a seeing-limited, wide-field multi-slit spectrograph. While conclusions from the first phase of these studies will feed into the design of the TMT facility much more work needs to be done before the NIR instrument concepts are competed and defined. 


\section{ACKNOWLEDGEMENTS}

The Thirty Meter Telescope (TMT) Project is a partnership of the Association of Universities for Research in Astronomy (AURA), the Association of Canadian Universities for Research in Astronomy (ACURA), the California Institute of Technology and the University of California. The partners gratefully acknowledge the support of the Gordon and Betty Moore Foundation, the US National Science Foundation, the National Research Council of Canada, the Natural Sciences and Engineering Research Council of Canada, and the Gemini Partnership. The author would also like to acknowledge the support from the other members of the TMT Instrumentation Working Group (David Crampton, Gordon Walker, David Sprayberry, Doug Simons, Steve Vogt, James Larkin and Keith Matthews) for fulsomely contributing to these deliberations and to Harland Epps, Joe Miller, Damien Jones, Denis Laurin, Ian Powell (and others at HIA) for their work and creative ideas. Finally I would like to acknowledge the pioneering work of Bev Oke. As far as I am aware, Bev was the first person to propose a seeing-limited instrument for CELT in 1999 and subsequently led the Canadian WFOS team until his sudden death in March 2004.

\section{REFERENCES}

1. J. Nelson + TMT SAC: “TMT Science Requirements Document (V13.2)” TMT document R-52. (May 2004).

2. R. Content: "New design for integral field spectroscopy with 8-m telescopes", Proc. S.P.I.E., 2871, pp. 1295-1305, (1997).

3. M.A. Kenworthy, I.R. Parry \& K. Taylor: "SPIRAL Phase A: A Prototype Integral Field Spectrograph for the $A A T$ ' PASP 113, p215, (2001).

4. R. Haynes, I.K. Baldry, K. Taylor \& D. Lee: "Characterisation of cooled infrared fibres for the Gemini IRMOS" Proc. S.P.I.E. 4008, pp. 1203-1214, (2000).

5. G. S. Wright, R. M. Sharples, P. R. Hastings, M. Wells, E. Atad-Ettedgui, J. R. Allington-Smith, D. J. Robertson,

6. R. Content, I. R. Parry, "GIRMOS - an infrared multi-object spectrograph for Gemini”, Proc, S.P.I.E. 4008, p729, (2000)

7. E. Prieto, J. Cuby, C. Lucuix, D. Ferrand, P. Blanc: “A new concept for feeding mulitple IFU and application for KMOS” Proc. S.P.I.E. 5492, paper 71 (2004)

8. A. McGrath, A. Moore: "Starbug - enabling the smart focal plane" Proc. S.P.I.E. 5492, paper 83 (2004)

9. K. Taylor, R.D. Cannon \& F.G. Watson: "The Anglo-Australian Observatory 2dF project" Proc.S.P.I.E., 2871, p145. (1997)

10. H.W. Epps: "Conceptual Description of an Efficient Large Verstile Imaging Spectrograph (ELVIS) for a 30-Meter Telescope (TMT)" UCO/Lick Observatory Technical Note. (March 2004)

11. B. Oke, D. Crampton, D. Laurin, J. Pazder, I. Powell, S. Roberts, K. Szeto: “A Canadian wide-field optical spectrograph for a 30-m telescope" Proc. S.P.I.E. 5492 paper 64 (2004) 